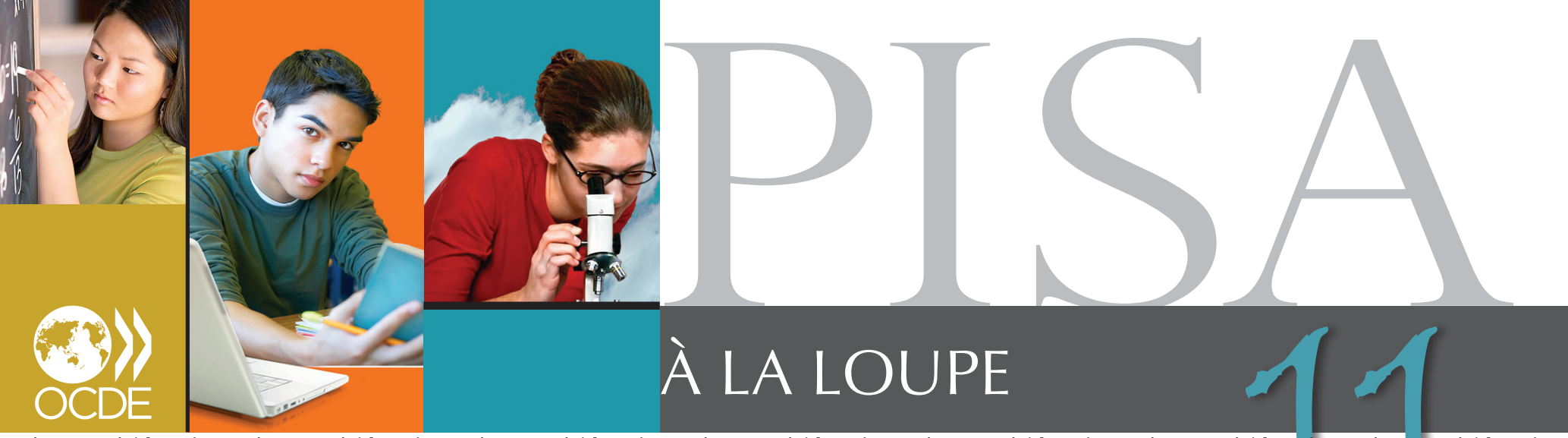

politiques d'éducation politiques d'éducation politiques d'éducation politiques d'éducation politiques d'éducation politiques d'éducation politiques d'éducation

\title{
Quelle est la réponse des systèmes d'éducation face à l'effectif croissant d'élèves issus de l'immigration?
}

- Dans les pays de l'OCDE, la proportion d'élèves issus de l'immigration a augmenté de 2 points de pourcentage, en moyenne, entre 2000 et 2009.

- Les élèves issus de l'immigration constituent plus de 5\% de l'effectif total des élèves dans 13 pays de l'OCDE et pays et économies partenaires ayant participé à l'enquête PISA 2009.

- Dans la plupart des pays, les élèves issus de l'immigration sont à la traîne par rapport aux élèves autochtones : dans de nombreux pays, l'écart de performance est même considérable. Cependant, l'Allemagne, l'Australie, la Belgique, le Canada, la Nouvelle-Zélande et la Suisse sont parvenus à réduire, voire à combler, cet écart.

Qu'il s'agisse de fuir des conflits, de concrétiser des espoirs d'une vie meilleure ou encore de profiter de possibilités sociales et économiques plus nombreuses, les hommes ont toujours traversé les frontières depuis que celles-ci existent. Au cours des prochaines décennies, la modernisation des moyens de transport et de communication, la mondialisation des marchés de l'emploi et le vieillissement des populations des pays de l'OCDE constitueront autant de facteurs déclencheurs de migrations. Le maintien de la cohésion sociale face à ces movements migratoires passera par l'intégration des immigrés et de leurs familles dans le pays d'adoption. À cet égard, l'éducation constitue un puissant moteur d'assimilation.

Les élèves autochtones sont ceux qui sont nés dans le pays de l'évaluation PISA ou dont au moins l'un des parents est né dans ce pays.

Les élèves issus de l immigration peuvent être dits de première génération (nés à l'étranger de parents nés à l'étranger) ou de deuxième génération (nés dans le pays de l'évaluation de parents nés à l'étranger). 
Pourcentage d'élèves issus de l'immigration en 2000 et 2009

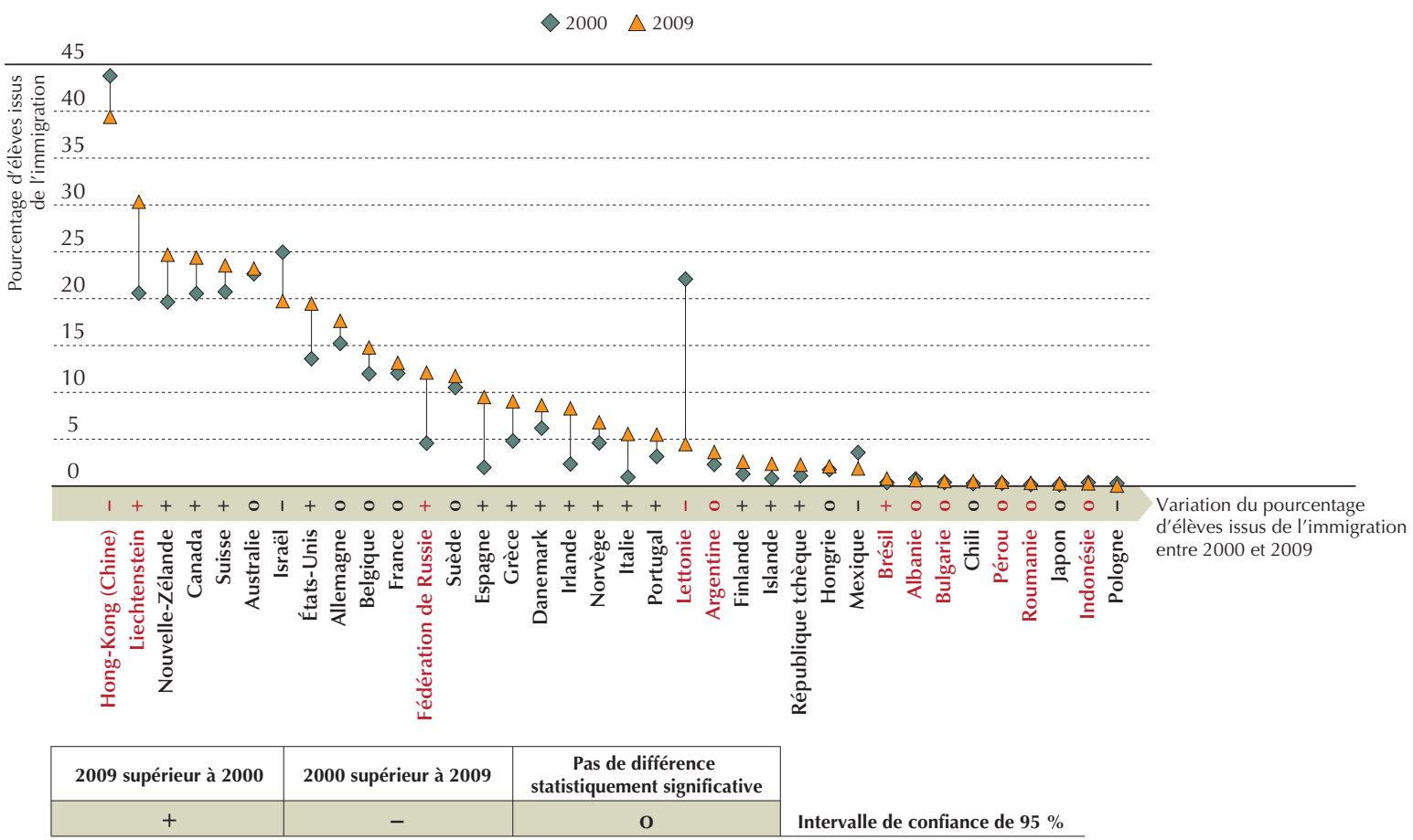

Les pays sont classés par ordre décroissant du pourcentage d'élèves issus de l'immigration en 2009. Source: Base de données PISA 2009 de l'OCDE.

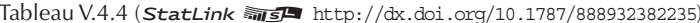

Figure V.4.6 (StatLink 青15 http://dx.doi.org/10.1787/888932360005).

La proportion d'élèves issus de l'immigration continue de croître...

Si l'on en croit les données collectées à partir des questionnaires administrés lors de l'enquête PISA 2009, le pourcentage d'élèves de 15 ans issus de l'immigration a augmenté en moyenne de 2 points de pourcentage entre 2000 et 2009 parmi les pays de l'OCDE dont les données sont comparables. Les élèves issus de l'immigration constituent à présent plus de $5 \%$ de l'effectif d'élèves de 15 ans dans 13 pays de l'OCDE et pays et économies partenaires. En Espagne, aux États-Unis, en Irlande et en Nouvelle-Zélande, et dans les pays partenaires en Fédération de Russie et au Liechtenstein, la proportion d'élèves issus de l'immigration a crû d'au moins 5 points de pourcentage au cours de la dernière décennie ; ceux-ci représentent aujourd'hui entre $8 \%$ et $30 \%$ des effectifs d'élèves de ces pays. En Italie, en Grèce et au Canada, le pourcentage d'élèves issus de l'immigration a connu une hausse de 3 à 5 points de pourcentage sur la même période. Près de $25 \%$ des élèves canadiens sont issus de l'immigration.
... mais dans certains pays,

l'écart de performance se comble.

Pourquoi ces chiffres sont-ils importants pour I'action publique en matière d'éducation ? Les conclusions des cycles PISA 2000 et PISA 2009 révèlent que si les élèves autochtones ont tendance à obtenir de meilleurs résultats que leurs homologues allochtones - avec un avantage de plus de 40 points en moyenne -, certains pays sont parvenus à combler l'écart de façon spectaculaire. Par exemple, en Belgique et en Suisse, l'écart de performance s'est réduit de près de 40 points sur cette période, même si les élèves autochtones obtiennent toujours de meilleurs résultats que les élèves issus de I'immigration, avec un avantage de 68 points en Belgique, et de 48 points en Suisse. Qui plus est, la Suisse a réussi à réduire l'écart de performance alors que le pourcentage d'élèves issus de l'immigration a augmenté durant cette même période. L'Allemagne, la Nouvelle-Zélande et dans les pays partenaires, le Liechtenstein, font également état d'un amenuisement de l'écart de performance entre ces deux groupes d'élèves. 


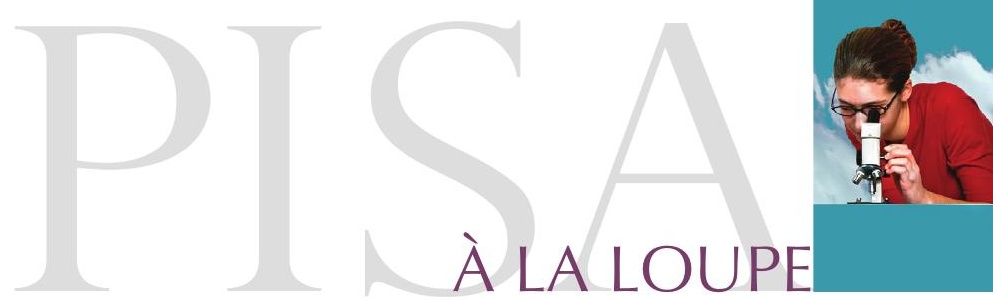

Performance en compréhension de l'écrit

selon I'ascendance autochtone ou allochtone,

avant et après prise en compte du milieu

Pourcentage

dère

valués par le PISA 2009

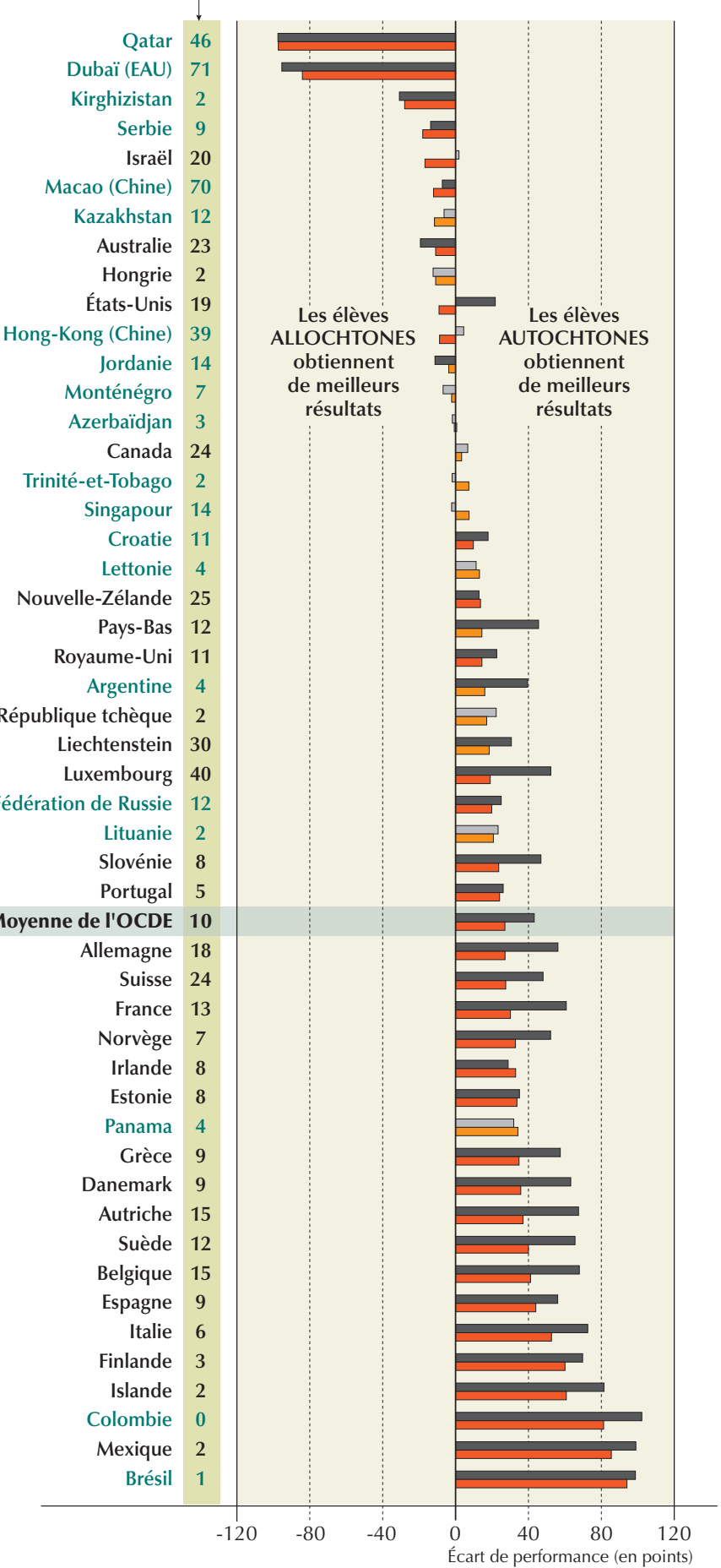

Certains systèmes d'éducation parviennent à réduire l'écart de performance entre tous les élèves, que ceux-ci soient ou non issus de l'immigration. En Australie par exemple, les élèves issus de l'immigration réussissent mieux que leurs camarades autochtones. Au Canada, les élèves issus de l'immigration ont aussi bien réussi que les élèves autochtones en 2009 ; ils constituent d'ailleurs une proportion importante, et en constante augmentation, de l'effectif d'élèves de ce pays.

\section{Le milieu socio-économique ne suffit pas à expliquer l'écart de performance.}

Ces tendances nous montrent qu'il existe plusieurs façons pour les pays et les établissements d'enseignement d'aider les élèves issus de l'immigration à surmonter certains des désavantages liés à ce statut. Souvent, les élèves issus de l'immigration sont défavorisés sur le plan socio-économique. En moyenne, dans les pays de l'OCDE, les parents de ces élèves ont un niveau de formation inférieur et occupent des emplois moins qualifiés que leurs pairs autochtones. Par ailleurs, les élèves issus de l'immigration ont tendance à avoir un accès réduit à des ressources éducatives et matérielles à la maison, par rapport à leurs camarades autochtones. Ainsi, à titre d'exemple, après contrôle du statut socio-économique des élèves au Luxembourg, l'écart de performance entre les élèves issus de l'immigration et les élèves autochtones se réduit de 52 à 19 points. En moyenne, dans les pays de l'OCDE, l'écart passe de 43 à 27 points lorsque l'on compare des élèves de milieux socio-économiques similaires, qu'ils soient ou non issus de l'immigration.

Cependant, le fait qu'un écart de performance équivalent à plus d'une demi-année de scolarité persiste même après prise en compte du milieu socio-économique suppose que d'autres facteurs entrent en jeu dans la performance des élèves. Dans une certaine mesure, ceux-ci pourraient être liés au lieu de naissance des élèves - par exemple s'ils sont immigrés de première ou de deuxième génération - ou à la langue parlée en famille - la langue de l'évaluation PISA ou bien une autre langue.

Remarque : les écarts de performance statistiquement significatifs sont indiqués en couleur plus foncée. Les pays sont classés par ordre croissant de l'écart de performance entre élèves autochtones et élèves allochtones, après prise en compte du milieu économique, social et cuturel des élèves. Source: Base de données PISA 2009 de l'OCDE.

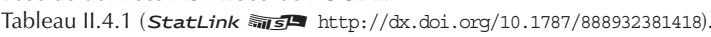
Figure II.4.5 (StatLink 蒚5 http://dx.doi.org/10.1787/888932343608). 
Élèves issus de l'immigration et performance en compréhension de l'écrit en 2000 et 2009

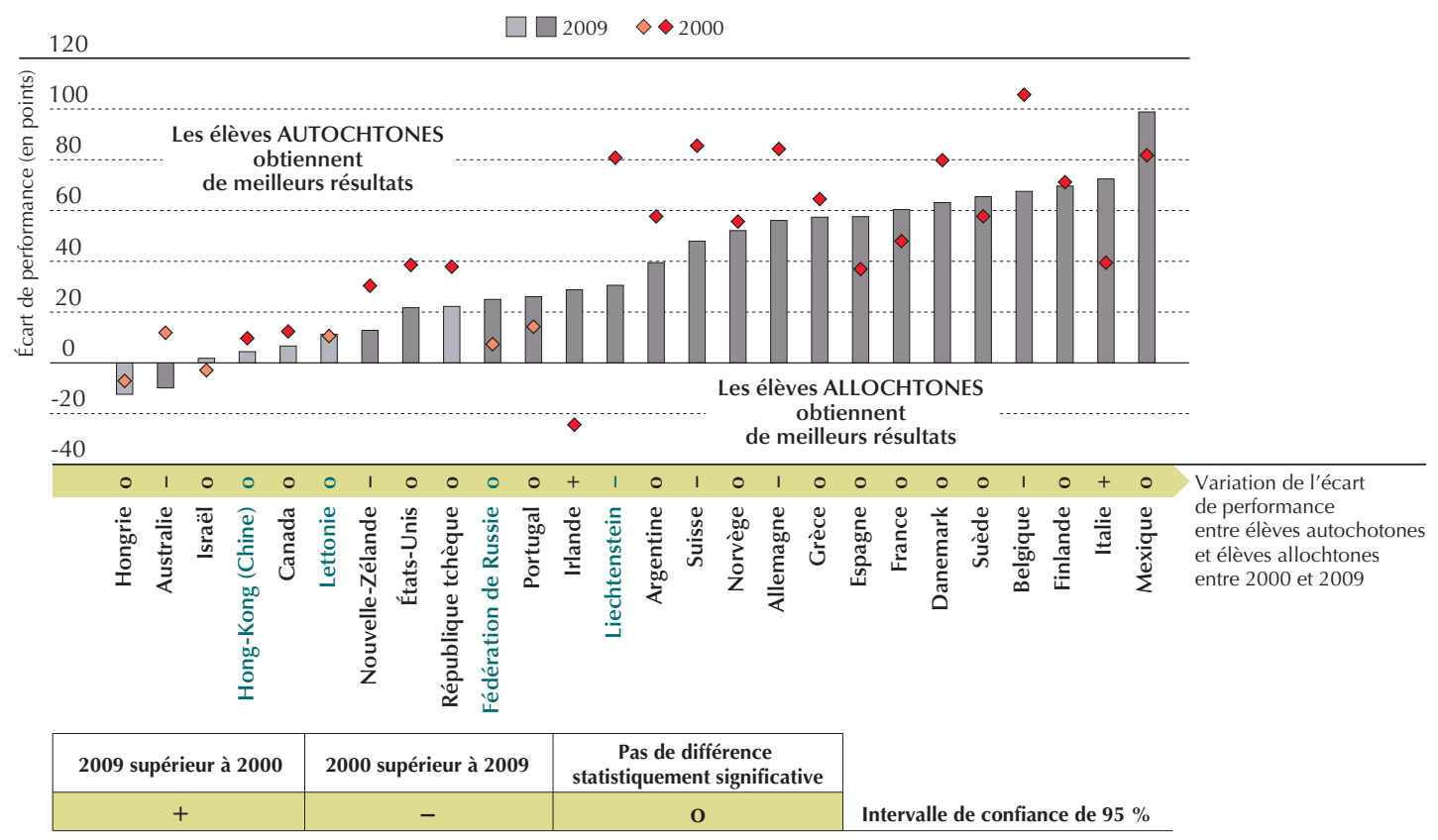

Remarque : les écarts de performance statistiquement significatifs sont indiqués en couleur plus foncée.

Les pays sont classés par ordre croissant de l'écart de performance entre élèves autochtones et élèves allochtones en 2009.

Source: Base de données PISA 2009 de l'OCDE.

Tableau V.4.4 (StatLink 解田 http://dx.doi.org/10.1787/888932382235).

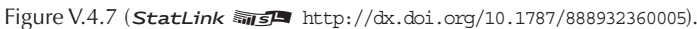

Toutefois, si l'on considère que l'écart de performance varie fortement d'un pays à l'autre - même en prenant en compte ces autres facteurs -, et étant donné que dans certains pays, cet écart de performance a beaucoup évolué dans le temps, il est clair que l'action publique peut permettre d'améliorer la situation. Par exemple, un enseignement efficace de la langue du pays d'accueil est crucial : les élèves qui ne comprennent pas leurs enseignants ne pourront rien apprendre d'eux.

Pour conclure : les systèmes d'éducation peuvent encourager une complète intégration sociale et économique de leurs élèves issus de l'immigration en identifiant les facteurs spécifiques qui empêchent ces élèves d'obtenir de bons résultats et en élaborant des programmes adaptés à leurs besoins.

Pour tout complément d'information

Contacter Maciej Jakubowski (Maciej.Jakubowski@oecd.org)

Consulter Résultats du PISA 2009 : Surmonter le milieu social : L'égalité des chances et l'équité du rendement de l'apprentissage (Volume II) et Résultats du PISA 2009: Tendances dans l'apprentissage : L'évolution de la performance des élèves depuis 2000 (Volume V)

\section{Voir}

www.pisa.oecd.org www.oecd.org/pisa/infocus
Prochain numéro

Garçons et filles sont-ils également prêts

à affronter l'ère numérique? 